\title{
Artículos
}

\section{Exposición al ruido por tráfico vehicular y su impacto sobre la calidad del sueño y el rendimiento en habitantes de zonas urbanas}

\section{Exposure to noise by vehicular traffic and impact on the quality of sleep and performance in residents of urban areas}

\author{
Benito Zamorano González \\ Yolanda Velázquez Narváez ${ }^{\mathrm{b}}$ \\ Fabiola Peña Cárdenas ${ }^{\mathrm{c}}$ \\ Lucía Ruiz Ramos ${ }^{\mathrm{d}}$ \\ Óscar Monreal Aranda ${ }^{\mathrm{e}}$ \\ Víctor Parra Sierra ${ }^{\mathrm{f}}$ \\ José Ignacio Vargas Martínez ${ }^{\mathrm{g}}$
}

\section{Resumen}

Los objetivos del estudio son: 1) determinar el nivel de ruido generado por vehículos automotores en la ciudad de Matamoros, Tamaulipas, y 2) identificar el impacto del ruido ambiental sobre la calidad del sueño y el rendimiento de los habitantes del área

${ }^{a}$ Universidad Autónoma de Tamaulipas, Unidad Multidisciplinaria Matamoros. Dirección: Calle Marte y Av. del Maestro, s.n., col. Alianza, Matamoros, Tamaulipas, México. Correo: bzamorano@docentes.uat.edu.mx ORCID: http://orcid.org/0000-0003-0422-3001

b Universidad Autónoma de Tamaulipas, Unidad Multidisciplinaria Matamoros, México. Correo: yovelazquez@docentes.uat.edu.mx ORCID: http://orcid.org/0000-0001-5591-8474

${ }^{\mathrm{c}}$ Universidad Autónoma de Tamaulipas, Unidad Multidisciplinaria Matamoros, México. Correo: fcardenas@docentes.uat.edu.mx ORCID: https://orcid.org/0000-0001-5895-0816

d Universidad Autónoma de Tamaulipas, Unidad Multidisciplinaria Matamoros, México. Correo: luruiz@docentes.uat.edu.mx ORCID: http://orcid.org/0000-0002-7122-6758

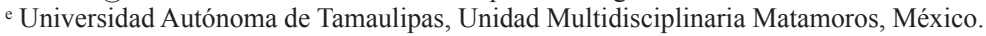
Correo: omonreal@uat.edu.mx ORCID: https://orcid.org/0000-0003-2039-6375

${ }^{\mathrm{f}}$ Universidad Autónoma de Tamaulipas, Unidad Multidisciplinaria Matamoros, México. Correo: vparra@docentes.uat.edu.mx ORCID: https://orcid.org/0000-0002-3877-9713

${ }^{\mathrm{g}}$ Universidad Autónoma de Tamaulipas, Unidad Multidisciplinaria Matamoros, México. Correo: jvargas@docentes.uat.edu.mx ORCID: https://orcid.org/0000-0003-2467-5806 
urbana. La investigación fue cuantitativa, transversal y correlacional. El ruido se midió en siete cruces, y para la recolección de datos sobre la calidad del sueño y el rendimiento; la muestra fue de 732 participantes. Se concluyó que el ruido vehicular se asocia a la calidad subjetiva del sueño, la cual, a su vez se relaciona significativamente con el rendimiento.

Palabras clave: ruido vehicular; calidad del sueño; rendimiento; zona urbana.

\begin{abstract}
The objectives of the study are: 1) to determine the level of noise generated by motor vehicles, at the city of Matamoros, Tamaulipas, and 2) to identify the impact of environmental noise on sleep quality and performance of the inhabitants of the urban area. The study was quantitative, transversal and correlational. Noise was measured in seven crossings, and for the collection of data on the quality of sleep and performance; the size of shows was of 732 participants. It was concluded that traffic noise is associated with the subjective sleep quality, which is significantly related to the performance.
\end{abstract}

Keywords: vehicular noise; quality of sleep; performance; urban area.

\title{
Introducción
}

Generalmente, cuando se habla de desarrollo urbano se piensa en los grandes beneficios que dicho desarrollo representa para la comodidad del ser humano. La inversión en infraestructura se supone sinónimo de accesibilidad, confort y beneficios económicos, pues las propiedades cercanas incrementan su plusvalía, los costos de traslado disminuyen, se ahorra tiempo en la realización de actividades cotidianas, entre otras ventajas. Muchas veces este crecimiento se presenta de forma tan acelerada, que para los mismos pobladores es difícil asimilarlo, y para cuando se dan cuenta, las zonas residenciales apacibles que habitaban, de pronto se ven rodeadas de grandes vialidades, con los beneficios e inconvenientes que de ello se derivan.

Uno de los impactos negativos que tiene el uso de vehículos automotores en las grandes ciudades es sobre el medio ambiente, incrementando la contaminación acústica. El ruido se define como "todo sonido indeseable que moleste o perjudique a las personas" (NOM-081-ECOL-1994: 4.26). En un sentido más amplio, de acuerdo con el Observatorio de Salud y Medio Ambiente de Andalucía (OSMAN, s.f.), se establece que el ruido se integra por dos componentes principales: el primero contempla el aspecto físico, y el segundo se considera de tipo subjetivo al referirse a la sensación de molestia que manifiesta el receptor. 
$\mathrm{Al}$ respecto, el Centro Estatal de Vigilancia Epidemiológica y Control de Enfermedades (CEVECE), asevera que el ruido debe considerarse como un problema ambiental de gran relevancia, además de ser un factor de riesgo sanitario. Así mismo, lo reconoce como un problema de salud pública que va en aumento debido al crecimiento poblacional y al incremento en la cantidad de medios de transporte público y privado.

En este sentido, Ramírez y Domínguez (2011, p. 510) afirman que en las zonas urbanas la principal fuente emisora de ruido ambiental es el transporte vehicular, debido al servicio que presta para la realización de actividades cotidianas, lo cual es característico de las ciudades modernas y conforma una problemática poco atendida en los países en vías de desarrollo. Por su parte, Martínez $(2005$, p. 2) asegura que el ruido por tráfico se encuentra asociado al incremento de vehículos automotores, impactando la vida de los habitantes; ello es reafirmado por Alfie y Salinas (2017, p. 68), quienes consideran que el ruido ambiental se encuentra asociado al uso de vehículos, los cuales lo generan a través de sus propios "mecanismos, motores y el roce de los neumáticos con el pavimento". Además, existen ruidos de tráfico independientes al vehículo, sobre todo en las zonas residenciales, que derivan principalmente del uso de altavoces o cláxones que son utilizados por el conductor para ofrecer un producto a la venta mientras se desplaza por las calles.

Se sabe que el ruido es un factor nocivo para la salud del ser humano. Sus efectos pueden clasificarse en dos grupos: físicos y psicosociales, aunque en muchas ocasiones se presentan efectos combinados. En lo concerniente a los efectos psicológicos, la OMS (1999, pp. 4-5) reconoce los relativos sobre la salud mental, el rendimiento, la conducta y la vida social, así como los trastornos del sueño. Al respecto, según De Santillana et al. (2010, p. 8), los trastornos del sueño presentan una elevada prevalencia en la población; se calcula que entre 20 y $30 \%$ de los pobladores de los países industrializados en Occidente lo padecen, lo cual propicia la aparición de otros factores psicosociales, tales como la disminución de la capacidad en el rendimiento laboral, el incremento de la probabilidad de ocurrencia de accidentes, y las repercusiones en las funciones cognitivas, como son las afecciones en la concentración en una tarea específica, en la productividad, en la conducta, y también en la calidad de la vida sexual. Es decir, los efectos del ruido van más allá de lo físico debido a las consecuencias a mediano y largo plazo que pueden observarse en los diferentes contextos del individuo. En este sentido, Álvarez (s.f.) establece que los efectos que el ruido ejerce sobre el comportamiento del individuo tienen que ver mayormente con su rendimiento y comportamiento social. Así mismo, se generan efectos psicosociales y se 
merma el proceso de comunicación entre las personas. Por lo tanto, el ruido es considerado como un factor potencial de riesgo para la seguridad al interferir en la comunicación y la concentración de quienes se exponen a él.

El sueño es una necesidad primaria para el hombre, tan relevante como la necesidad de beber o comer, y dicha necesidad puede llegar a ser más imperiosa que otras de carácter biológico (Chóliz, 1994, p. 217). De la misma forma, Hernández (2006) denomina al sueño como un "proceso fisiológico necesario" que desempeña un importante papel en el "desarrollo precoz del cerebro, en el aprendizaje y en la consolidación de la memoria".

En este sentido, Medina, Feria y $\operatorname{Oscoz}(2009$, p. 2) reconocen dos fases del sueño. La primera contempla el sueño REM (Rapid Eye Movement) o sueño onírico, que es la etapa donde el cerebro permanece activo. La segunda fase corresponde al sueño no REM o NREM, el cual se encuentra dividido en cuatro etapas, en las que de manera progresiva va tomando mayor profundidad. Para que el sueño se considere reparador debe repetirse un patrón donde el NREM es seguido por el REM durante aproximadamente 90 minutos cada uno, repitiéndose de cuatro a seis veces durante el ciclo del sueño, el cual generalmente tiene una duración de ocho horas. Por lo tanto, si una persona despierta antes de completar su ciclo normal de sueño, será mayor la probabilidad de que su descanso no sea reparador.

Sierra, Jiménez y Martín (2002, p. 38) coinciden en que los trastornos del sueño no sólo afectan la salud de la persona que los padece, sino su calidad de vida, que a su vez interfiere con la capacidad de atención sobre una tarea. Carskadon (1990, p. 5) identifica como consecuencias del sueño insuficiente en adolescentes, la somnolencia diurna, la mayor vulnerabilidad a tener accidentes, los cambios en el estado de ánimo, los problemas de conducta, el incremento en las probabilidades de consumir drogas y alcohol, así como la aparición de los principales trastornos del ciclo sueño-vigilia.

Existen múltiples factores que alteran esta actividad y generan una serie de consecuencias en la salud de las personas, los cuales son también conocidos como trastornos del sueño y se caracterizan por incidir negativamente sobre la calidad del mismo. En este sentido, Blaivas (2012) menciona que existen más de cien trastornos diferentes del sueño, los cuales pueden agruparse en cuatro categorías: insomnio, somnolencia diurna excesiva, trastornos del ritmo circadiano y conductas anormales durante el sueño. Por otra parte, los trastornos del sueño pueden clasificarse como orgánicos y no orgánicos. Los primeros se manifiestan como sintomatología de un padecimiento patológico, mientras que los segundos tienen que ver con factores externos que impactan sobre la calidad del sueño. Dentro de esta clasificación de factores externos se encuentra la exposición al ruido. 
De acuerdo con Bobes (1992, p. 100), el umbral de ruido que es necesario para ocasionar un despertar repentino dependerá de la fase del sueño y de la necesidad de sueño de la persona; sin embargo, "nunca llega a existir una adaptación a un nivel alto de ruido". Los ruidos que mayormente afectarán son aquellos que son intensos y ocasionales. En este sentido, se pueden identificar algunos ruidos producidos por el tráfico vehicular con estas características, como pudiera ser el sonido de un claxon, la música a nivel alto de un coche que pasa, el rechinido de llantas, entre otros. Así mismo, el autor asegura que a menor nivel de ruido, la calidad del sueño es mejor.

Por otra parte, Chávez (2006, p. 43) señala que los efectos que el ruido ejerce sobre el sueño pueden clasificarse en tres principales grupos. El primero se relaciona con las interferencias que este factor de riesgo tiene sobre el sueño, por ejemplo, la dificultad para conciliarlo o las interrupciones durante el periodo de sueño. Así mismo, se incluyen en este grupo algunas manifestaciones físicas como el incremento de la presión arterial o del ritmo cardíaco, los cambios en la frecuencia respiratoria, los movimientos corporales, entre otros. El segundo grupo incluye los efectos posteriores a la exposición al ruido, como la fatiga, las modificaciones en la conducta y/o la alteración del bienestar. Como tercer grupo, el autor identifica los efectos a largo plazo, refiriéndose a la aparición de enfermedades orgánicas producidas por las alteraciones del sueño.

Existen diversos estudios que ponen de manifiesto la relación entre el ruido del tráfico y la calidad subjetiva del sueño. En un estudio realizado por Sygna, Aasvang, Aamodt, Oftedal y Hjertager (2014, p. 17), en una muestra de 2898 participantes, se encontró que los individuos que presentaron una calidad del sueño pobre pueden ser más vulnerables a los efectos del ruido del tráfico sobre su salud mental, en comparación con los que presentaron una mejor calidad del sueño. Por otra parte, Freir, Mohler y Röösli (2014, p. 194) encontraron que, en una muestra de 1122 personas, los efectos del ruido del tráfico sobre la calidad objetiva del sueño fueron independientes a la percepción de molestias por el ruido, mientras que la asociación entre la calidad subjetiva del sueño y el ruido se encuentra mediada por la percepción de molestias por ruido. En otro estudio, realizado por Öhrström, Hadzibajramovic, Holmes y Svensson (2006, p. 116), en una población conformada por 160 padres de familia y 160 menores de 9 a 12 años de edad, se encontró que en ambos grupos los resultados mostraron que existe una relación entre los niveles de ruido del tráfico y la calidad del sueño, mientras que en los niños otro factor asociado al ruido vehicular es la somnolencia diurna. En este sentido, Quevedo y Quevedo (2011, p. 60) encontraron que existe una 
influencia significativa de los patrones de sueño sobre el rendimiento académico de la población estudiantil. Al respecto, Falleti, Maruff, Collie, Darby y McStephen (2003, p. 265) señalan que, de acuerdo con los resultados de su estudio, la fatiga o somnolencia diurna causa tanto deterioro como el alcohol respecto a la capacidad de atención continua y la memoria, y afecta al desarrollo del aprendizaje y a la precisión para realizar actividades complejas.

Por lo anterior, se considera pertinente el abordaje de esta problemática común entre los habitantes de las zonas urbanas. Para ello, el presente trabajo de investigación tiene dos objetivos principales: 1) determinar el nivel de ruido ambiental generado por el tránsito de vehículos automotores en las principales avenidas de la ciudad de Matamoros, Tamaulipas, México y, 2) identificar el impacto que tiene el ruido ambiental generado por el tránsito de vehículos automotores sobre la calidad del sueño y el rendimiento de los habitantes de la zona urbana.

\section{Contexto del estudio}

El presente trabajo de investigación se desarrolla en la zona urbana de la ciudad de Matamoros, ubicada en el estado de Tamaulipas, en la frontera norte de México (véase el Mapa 1).

Matamoros cuenta con 520367 habitantes, de acuerdo con cifras del último censo poblacional realizado por el Instituto Nacional de Estadística y Geografía (INEGI, 2015). De los 43 municipios que integran la entidad, Matamoros ocupa el segundo lugar respecto a la cantidad de habitantes, superado únicamente por Reynosa. La principal actividad económica corresponde a la industria maquiladora de exportación (IME), además de ser un importante cruce fronterizo comercial. Respecto a las características de la población, debe tenerse en cuenta que, si bien la circulación vial tiende a disminuir durante la noche, existe un grupo de trabajadores nocturnos. Aunque no se cuenta con cifras oficiales respecto a la cantidad de personas que componen dicho grupo, ya que la principal actividad económica de la zona es la industria maquiladora de exportación, la sobreoferta de fuerza laboral genera en las empresas la expectativa de que el trabajador "pueda movilizarse prácticamente a cualquier hora del año para la producción" (Carrillo, 1994, p. 21), generando los turnos nocturnos que aseguran que la producción no se detenga, sin importar el horario. Esto sin contar con los trabajadores de establecimientos de recreación y entretenimiento, áreas de salud, vigilancia y comercio. 


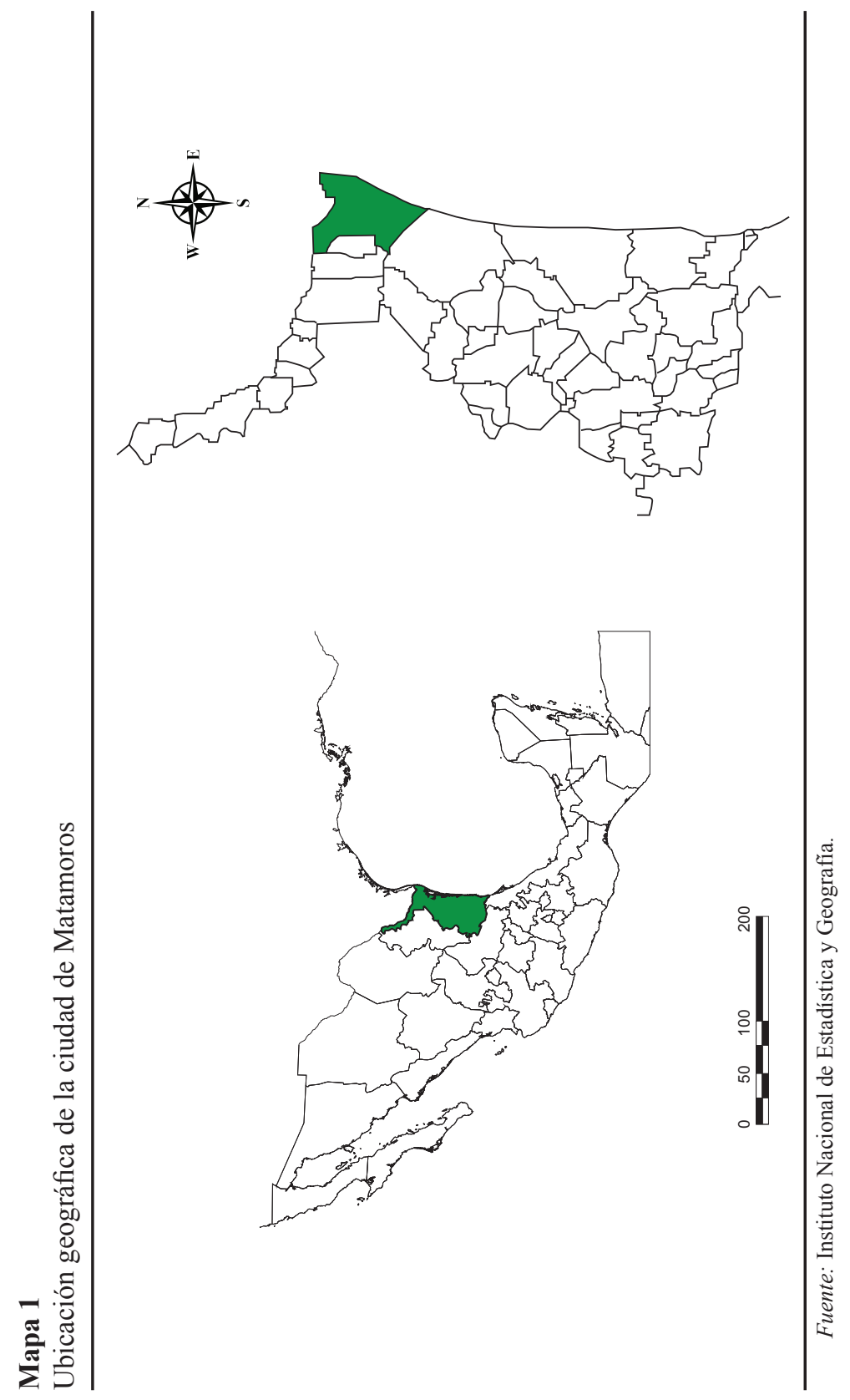


Esta circunstancia hace imprescindible que el periodo de sueño de un trabajador nocturno se realice durante el día. De acuerdo con Medina (2004, pp. 55-56), el 60\% de los trabajadores que laboran por la noche sufren alteraciones del sueño, ya que éstos suelen tener menos horas de sueño que los que trabajan durante el día (aproximadamente de cinco a seis horas). De la misma forma, el dormir en turno diurno influye sobre la profundidad del sueño. Esto es debido a diversos factores, incluyendo aquellos de índole ambiental, entre los que se encuentra el ruido. Al respecto, la Organización Mundial de la Salud (OMS, 1999, p. 8) también hace una distinción horaria al recomendar límites máximos de ruido ambiental, de tal forma que durante el día el ruido continuo exterior no debe rebasar los $55 \mathrm{~dB}(\mathrm{~A})$, o $50 \mathrm{~dB}(\mathrm{~A})$ para ruido moderadamente molesto. También considera que, para un descanso nocturno adecuado, el nivel de ruido continuo de fondo no debe exceder $\operatorname{los} 30 \mathrm{~dB}(\mathrm{~A})$, por considerarse que a partir de este valor inician los efectos cuantificables del ruido sobre el sueño. Así mismo, para sucesos de ruido únicos el valor máximo no debe ser superior a $45 \mathrm{~dB}(\mathrm{~A})$.

En lo concerniente a las características del parque vehicular, al ser una ciudad fronteriza, es común la circulación de transportes comerciales que se dirigen a Estados Unidos, o ingresan productos y/o materias primas al país. En este sentido, Matamoros se conoce como "la gran puerta de México", haciendo alusión a esta actividad transfronteriza.

Otra característica relacionada al tráfico de la ciudad es "la permanente presencia de tráfico vehicular extranjero e irregular del cual no existe registro oficial" (Velázquez, Zamorano y Ruiz, 2017, p. 10). Al respecto, el Instituto Nacional de Ecología y Cambio Climático (2010, citado por Zamorano, Peña, Parra, Velázquez y Vargas, 2015, p. 22) ha considerado que la cantidad de vehículos registrados ante la Secretaría de Finanzas es de 76 533. Sin embargo, de acuerdo con el "Reporte final de emisiones y parque vehicular de Matamoros y Reynosa, Tamaulipas", la cantidad de vehículos activos asciende a 267 290. Esto representa una diferencia de casi 200000 vehículos.

Es un hecho que, debido a esta problemática, resulta imposible dar una cifra exacta de los vehículos activos. Lo que sí se puede afirmar es que el tránsito vehicular representa un problema en aumento respecto al ruido que genera, ya que, por tratarse de un país en vías de desarrollo, los vehículos suelen ser antiguos y son adquiridos a bajo costo por ser de segunda mano, lo que significa que en muchas ocasiones las condiciones mismas del automóvil agravan la emisión de ruido al circular por las calles de la ciudad.

En México existen disposiciones legales en materia de prevención de riesgos asociados a exposiciones a ruido, generadas por diferentes depen- 
dencias. Estas regulaciones establecen criterios máximos de emisión y exposición a niveles de ruido para evitar los daños a la salud que pudieran generarse de una sobreexposición. Estas disposiciones pueden dividirse en dos: 1) las que garantizan la protección de la población con exposición laboral al ruido y, 2) las que regulan la generación de ruido ambiental que puede afectar la salud de la comunidad en general.

En cuanto a la emisión de ruido ambiental, éste se clasifica de acuerdo al tipo de fuente que lo genera: 1) fuentes móviles y 2) fuentes fijas. Respecto a las fuentes móviles, el Reglamento para la Prevención y Control de la Contaminación Ambiental Originada por la Emisión de Ruidos (1976, art. 32) establece los límites máximos de emisión de ruido ambiental, segregándolo en dos tipos de fuentes, como se observa en el Cuadro 1 y en el Cuadro 2.

Sin embargo, se hace notar que este reglamento no ha sido modificado desde el año 1976, por lo que es probable que los niveles máximos permitidos de emisión de ruido ambiental debieran ser evaluados nuevamente en relación con las características de los modelos de los automóviles que predominan en la actualidad. Para ello, en el ámbito internacional se ha empleado la Norma ISO 1996 (International Organization for Standarization, 2007 y 2016), denominada de forma general “Acústica. Descripción, medición y evaluación del ruido ambiental", la cual está estructurada en dos partes: en la primera se describen las magnitudes básicas y los métodos de evaluación del ruido medioambiental; la segunda se centra en la determinación de los niveles de presión acústica y su incertidumbre asociada. Con éstas se pretende establecer elementos para que las autoridades puedan realizar la medición y la evaluación del ruido en ambientes comunitarios, pudiéndose desarrollar normas nacionales y reglamentos, así como los correspondientes límites de ruido aceptables en la evaluación (ISO, 2007, 2016).

\section{Cuadro 1}

Niveles máximos permitidos de emisión de ruido ambiental por fuentes móviles (vehículos automotores), en $\mathrm{dB}(\mathrm{A})$

\begin{tabular}{|c|c|c|c|c|}
\hline \multirow[b]{2}{*}{ Año / modelo } & \multicolumn{4}{|c|}{ Peso bruto vehicular } \\
\hline & $\begin{array}{c}\text { Hasta } \\
2727 \mathrm{~kg}\end{array}$ & $\begin{array}{c}\text { De } 2728 \\
\text { a } 3500 \mathrm{~kg}\end{array}$ & $\begin{array}{c}\text { De } 3501 \\
a 11001 \mathrm{~kg}\end{array}$ & $\begin{array}{l}\text { Más de } \\
11001 \mathrm{~kg}\end{array}$ \\
\hline Anteriores a 1977 & 86 & 88 & 91 & 98 \\
\hline 1977 у 1978 & 83 & 85 & 88 & 95 \\
\hline 1979 en adelante & 80 & 83 & 85 & 92 \\
\hline
\end{tabular}

Fuente: Reglamento para la Prevención y Control de la Contaminación Ambiental Originada por la Emisión de Ruidos (1976, Art. 32). 


\section{Cuadro 2}

Niveles máximos permitidos de emisión de ruido ambiental por fuentes móviles (motocicletas, bicicletas y triciclos motorizados)

\begin{tabular}{lc}
\hline Año / modelo & Nivel máximo permitido en $\mathbf{d B}(\boldsymbol{A})$ \\
\hline Anterior a 1968 & 93 \\
1968 a 1975 & 92 \\
1976 a 1978 & 89 \\
1979 en adelante & 84 \\
\hline
\end{tabular}

Fuente: Reglamento para la Prevención y Control de la Contaminación Ambiental Originada por la Emisión de Ruidos (1976, Art. 32).

\section{Metodología}

El presente estudio tiene un diseño cuantitativo, correlacional y transversal. Fue desarrollado en la ciudad de Matamoros (Tamaulipas, México), la cual tiene una extensión geográfica de $4045.62 \mathrm{~km}^{2}$ y un total de 520367 habitantes, de los cuales el $51.3 \%$ son del género femenino y $48.7 \%$ varones (INEGI, 2015). Matamoros, por ser una ciudad fronteriza, tiene condiciones migratorias específicas que impactan sobre la cantidad de vehículos que circulan por sus vías. El registro vehicular oficial indica que existe un total de 132938 vehículos automotores; sin embargo, tal cantidad, al igual que el número total de la población, podría llegar a triplicarse debido a dichas características.

Para la obtención de datos de campo, el estudio se dividió en dos fases. La primera consistió en la medición de los niveles de ruido asociados a la circulación de los vehículos automotores; la segunda se concentró en tres variables en la población expuesta al ruido por el tráfico vehicular: 1) la calidad subjetiva del sueño, 2) la presencia de problemas para dormir a causa del ruido ambiental exterior percibido y, 3) el rendimiento, entendido como la disfunción durante el día. Estas fases se detallan a continuación.

\section{Fase 1. Medición de los niveles de ruido ambiental generado por el tráfico vehicular}

Para la medición del ruido ambiental generado por el tráfico vehicular se determinaron como áreas críticas las que presentaran la mayor afluencia vial, por lo que de manera intencional se realizó una preselección de 13 cruceros 
vehiculares. Ello permitió la ubicación de las calles y avenidas de mayor circulación en la ciudad. Estas vías son reconocidas por la Secretaría de Comunicaciones y Transportes (SCT) como arterias alimentadoras, con base en sus características de trayectos cortos y una velocidad promedio de $60 \mathrm{~km} / \mathrm{h}$ (Secretaría de Comunicaciones y Transportes, 1984, p. 6). Las características respecto al uso de suelo son principalmente comerciales y habitacionales.

Los valores del flujo vehicular fueron recopilados mediante el uso de un radar marca Safe Pace, el cual fue ubicado en los cruces a monitorear de forma no intrusiva, y se instaló en los soportes de las luminarias a una altura de tres metros. El equipo utilizado permitió la transferencia de los datos a una computadora mediante bluetooth al software SP-Data de la compañía Traffic Logix. De esta forma, se eligieron siete arterias alimentadoras, descartando tres vías colectoras y tres más por estar en reparación, las cuales se muestran en el Cuadro 3.

Una vez determinados los cruces, se procedió a la medición del ruido. Dichas mediciones fueron realizadas de lunes a viernes en tres periodos diferentes del día, contemplando un horario entre las 6.30 y las 18.30 horas. La manera de realizar las mediciones consideró que cada periodo tuviera una duración de una hora, desarrollándose en subperiodos de 15 minutos, lo que permitió el traslado a las diferentes aceras del cruce donde se realizó la evaluación. Debido a que no existe legislación mexicana que establezca un método para evaluar el ruido generado por el tráfico vehicular, se tomó como base la Norma ISO 1996-1 referente a la acústica, descripción, medición y evaluación del ruido ambiental: magnitudes básicas y métodos de evaluación (ISO, 2016, p. 5), así como también la Norma ISO 1996-2 (ISO, 2007, p. 1).

El equipo utilizado consistió en tres sonómetros tipo I, con sus respectivos calibradores de campo, de la marca Quest Technologies 3M. La instalación de éstos fue al menos a tres metros de distancia de cualquier fachada que pudiera reflejar el sonido y una altura de un metro y medio. Estos criterios se determinaron considerando que en la ciudad no predominan los edificios y construcciones de gran altura; también por cuestión logística en la medición, lo que implicaba realizar traslados en periodos de 15 minutos. El parámetro obtenido fue el nivel sonoro continuo equivalente $\left(\mathrm{L}_{\mathrm{eq}}\right)$, procediéndose con éste a determinar las incertidumbres estándar y expandida.

Con la finalidad de evitar un sesgo en la obtención de los niveles de ruido, se verificó que las condiciones ambientales predominantes contaran con las siguientes características: velocidad del viento menor a tres metros por segundo, y ausencia de temperaturas congelantes, lluvia y truenos.

Una vez obtenidos los resultados, se vaciaron a una computadora para su interpretación, utilizando el software QuestSuite Pro II. Es importante 


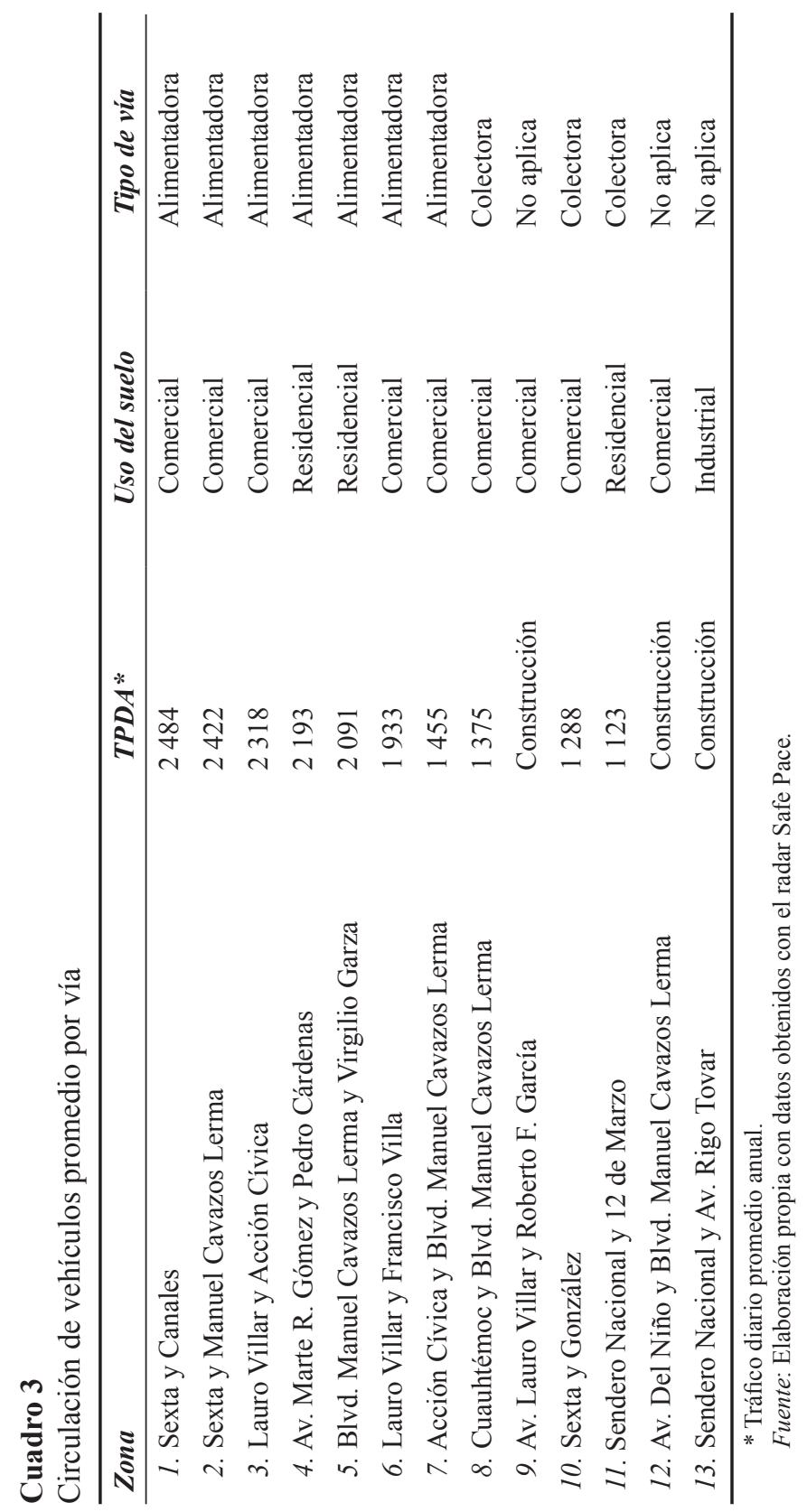


señalar que durante esta etapa se presentó la imposibilidad de aislar completamente el ruido generado por el paso de los vehículos automotores, del resto de los ruidos ambientales, como pueden ser los producidos por animales, vendedores, y por el tránsito de peatones, entre otros. Sin embargo, por la ubicación de los instrumentos de medición en relación a la dirección del viento, y los tiempos programados para la recolección de datos, puede afirmarse que el ruido generado por el tráfico vehicular se encuentra representado significativamente en los resultados.

\section{Fase 2. Medición de la calidad subjetiva del sueño, los problemas para dormir por el ruido ambiental exterior percibido y el rendimiento en la población expuesta al ruido generado por el tráfico vehicular}

Para la medición de las variables calidad subjetiva del sueño, problemas para dormir por causa de ruido ambiental exterior percibido y rendimiento, en la población expuesta al ruido por tráfico vehicular se contó con la colaboración de 732 participantes. La muestra fue seleccionada de forma no probabilística y por conveniencia. Las características de inclusión fueron: 1) habitar dentro del diámetro de un kilómetro de la zona determinada como crítica (contemplada en el Mapa 2), 2) participar voluntariamente y, 3) ser mayor de edad.

Previo a la aplicación del instrumento, cada participante fue notificado del objetivo del estudio a través de un consentimiento informado, mismo que establece un compromiso de confidencialidad por parte de los investigadores, garantizando el anonimato de los integrantes de la muestra. El instrumento utilizado para la recolección de los datos fue una adaptación del cuestionario de Pittsburg de calidad del sueño (PSQI), el cual quedó compuesto por 19 ítems agrupados en nueve preguntas, tres de las cuales son de respuesta abierta, una cerrada y seis en escala de Likert. Además de los niveles de ruido, en el presente estudio se consideraron principalmente tres variables: 1) calidad subjetiva del sueño, 2) problemas para dormir a causa del ruido ambiental exterior percibido, y 3) rendimiento. La confiabilidad del instrumento se obtuvo a través del coeficiente Alfa de Cronbach, alcanzando un valor de 0.746 . 


\section{Mapa 2}

Ubicación de los puntos de medición de ruido y tráfico vehicular

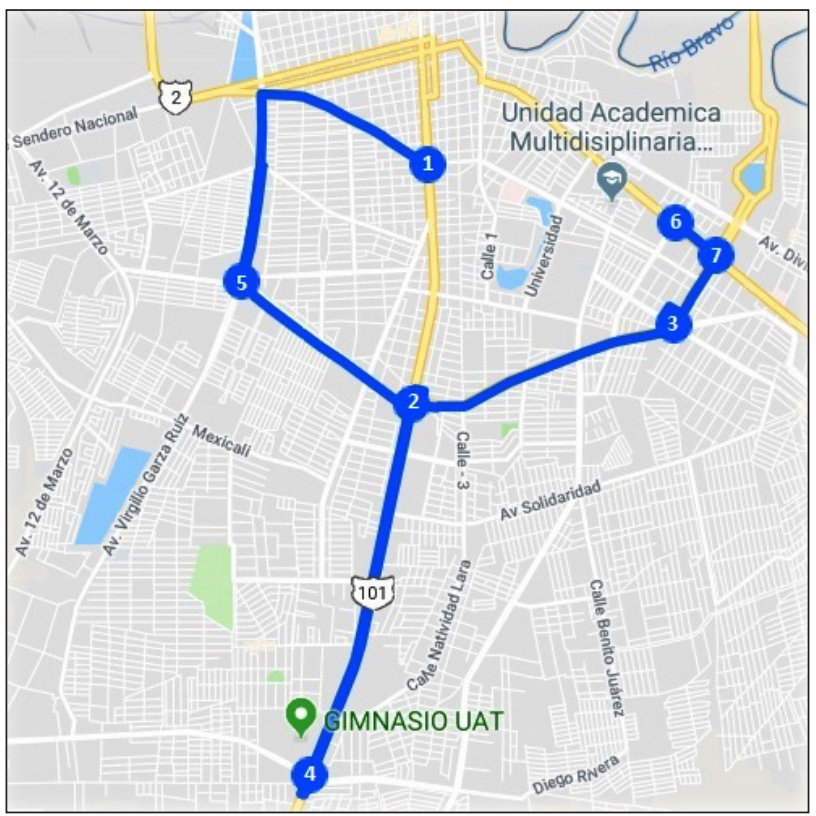

Fuente: Google Maps.

\section{Resultados}

\section{Resultados de la fase 1. Nivel de ruido ambiental generado por el tráfico vehicular}

Los resultados de los niveles de ruido identificados en las zonas evaluadas se muestran en el Cuadro 4. Como se aprecia en éstos, el nivel sonoro continuo equivalente $\left(\mathrm{L}_{\mathrm{eq}}\right)$ identificado en la mayoría de las zonas excede los 70 dB. La incertidumbre estándar se encuentra por debajo de los $\pm 2 \mathrm{~dB}$, mientras que la expandida en ningún caso rebasa los $\pm 3 \mathrm{~dB}$. A pesar de que la relación entre el tráfico vehicular y el nivel de ruido arroja un índice de correlación de Pearson 0.72, se presentó un p-valor de 0.067 . 


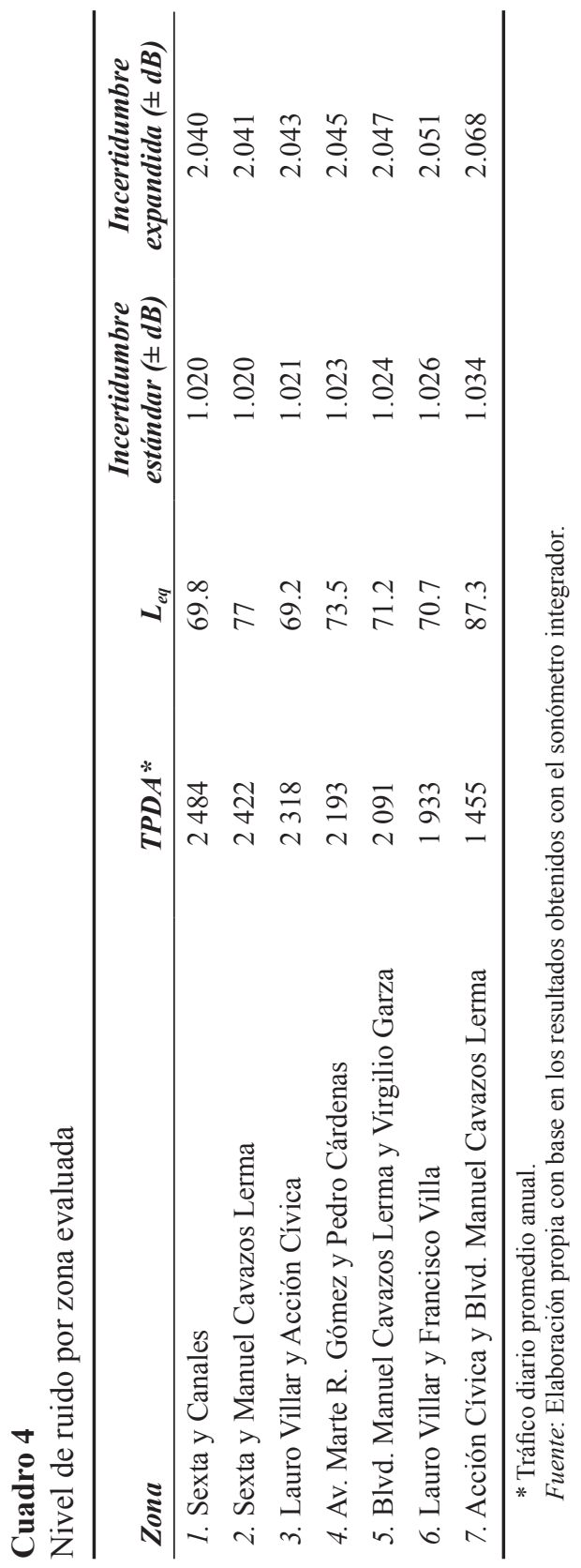


Resultados de la fase 2. Medición de la calidad subjetiva del sueño, los problemas para dormir por el ruido ambiental exterior percibido y el rendimiento en la población expuesta al ruido generado por el tráfico vehicular

\section{Distribución de la muestra}

La muestra estuvo conformada por personas de 18 a 71 años de edad. El 49\% tiene una edad dentro de un rango de 18 a 35 años, mientras que el 51\% cuenta con 36 años de edad o más. Respecto al género, el 47\% de los integrantes de la muestra son varones y el 51\% son mujeres.

En cuanto a la distribución de la muestra por cruce vial, los resultados se muestran en el Cuadro 5.

Relación entre las variables de estudio

El 55.3\% de la muestra, correspondiente a 405 sujetos, aseguró haber tenido problemas para dormir por lo menos una vez en el último mes, de los cuales el $81.4 \%$ identificó como causa principal el ruido ambiental exterior. De ellos, el 72\% identificó como fuente de dicho ruido el tráfico vehicular.

El 36\% de las personas encuestadas calificó de mala a muy mala su calidad de sueño, mientras que el $26.8 \%$ consideró que la disfunción durante el día (somnolencia y falta de ánimo) afecta su rendimiento en las actividades cotidianas.

\section{Cuadro 5}

Distribución de la muestra por cruce vial

\begin{tabular}{lccc}
\hline Cruce & $\boldsymbol{F}$ & $\boldsymbol{\%}$ & $\begin{array}{c}\boldsymbol{\%} \\
\text { acumulado }\end{array}$ \\
\hline Acción Cívica y Blvd. Manuel Cavazos Lerma & 188 & 25.7 & 25.7 \\
Lauro Villar y Acción Cívica & 111 & 15.2 & 40.8 \\
Lauro Villar y Francisco Villa & 100 & 13.7 & 54.5 \\
Sexta y Canales & 100 & 13.7 & 68.2 \\
Sexta y Manuel Cavazos Lerma & 100 & 13.7 & 81.8 \\
Blvd. Manuel Cavazos Lerma y Virgilio Garza & 100 & 13.7 & 95.5 \\
Av. Marte R. Gómez y Pedro Cárdenas & 33 & 4.5 & 100 \\
Total & 732 & 100 & \\
\hline
\end{tabular}

Fuente: Elaboración propia con los resultados del análisis de datos. 
En el Cuadro 6 se muestran los resultados de la correlación entre las variables de estudio.

Las correlaciones significativas se presentaron entre las variables mostradas en el Cuadro 7.

\section{Discusión}

El ruido ambiental asociado a la circulación de vehículos automotores en la ciudad de Matamoros (Tamaulipas, México), identificado en los siete cruces considerados en el presente estudio, rebasa los $69 \mathrm{~dB}(\mathrm{~A})$. Esto significa que dichos niveles de ruido exceden considerablemente la recomendación de la OMS (1999, p. 8), que señala límites para el ruido ambiental de 55 dB(A) durante el día y de $45 \mathrm{~dB}(\mathrm{~A})$ durante la noche en zonas habitacionales.

En este sentido, se debe tomar en cuenta que estos niveles corresponden al ruido que genera el vehículo por sus características inherentes, así como a otros factores asociados, como pueden ser el roce de las llantas con el pavimento, las condiciones de la vía, el uso del claxon, el exceso de velocidad, entre otros, que influyen en la cantidad de decibeles que se generan, según lo señalan Alfie y Salinas (2017, p. 68) y Morales (2006, p. A45).

Al respecto, aunque el Reglamento para la Prevención y Control de la Contaminación Ambiental Originada por la Emisión de Ruidos (1976, Art. 32) establece de 80 a $92 \mathrm{~dB}(\mathrm{~A})$ como límite máximo de emisión de ruido generado por vehículos automotores, para vehículos de modelos posteriores a 1979 sería irresponsable afirmar que existe cumplimiento normativo ya que, a pesar de no contar con cifras oficiales de la cantidad de vehículos que circulan por la ciudad de acuerdo al modelo-año, el Estudio de emisiones y actividad vehicular en Matamoros y Reynosa, del Instituto Nacional de Ecología y Cambio Climático (INECC, 2010, p. 8), señala que únicamente el $7.7 \%$ de una muestra compuesta por 12714 vehículos cuenta con una antigüedad de 20 años o más en relación al año 2009. Esto significa que los límites máximos permisibles de emisión de ruido, que marca actualmente dicho reglamento, deberían ser actualizados en función de las características de los modelos de los vehículos automotores que hoy en día predominan en las vías.

Lo anterior hace notar la necesidad que existe en México en cuanto a la regulación de la emisión de ruido ambiental por fuentes móviles, ya que la normatividad existente en materia de ruido considera parámetros que no contemplan el ruido generado por vehículos automotores en circulación (NOM -011-STPS 2001; NOM-079-ECOL-1994; NOM-080-ECOL-1994 y NOM-081-ECOL-1994). 


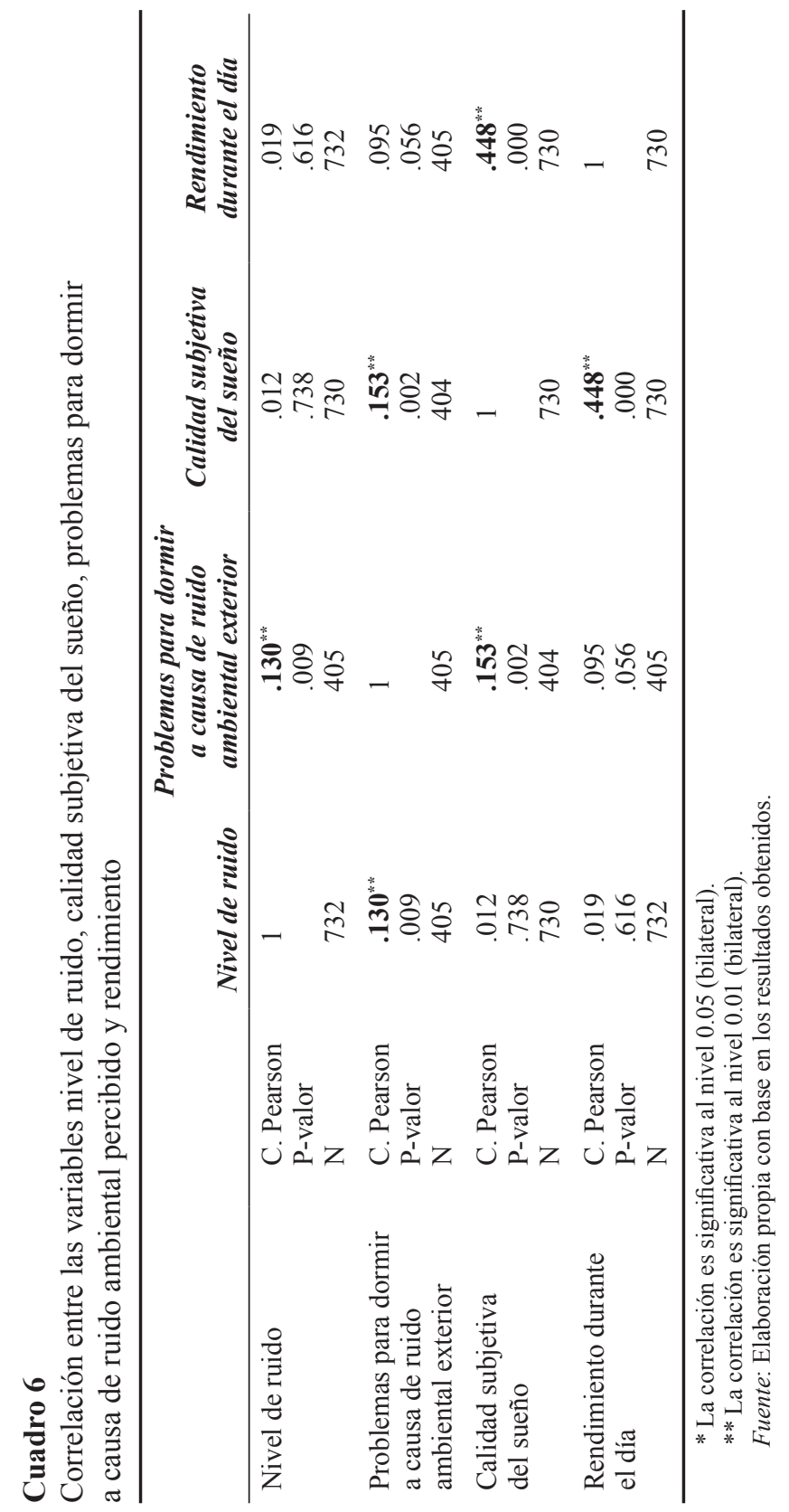




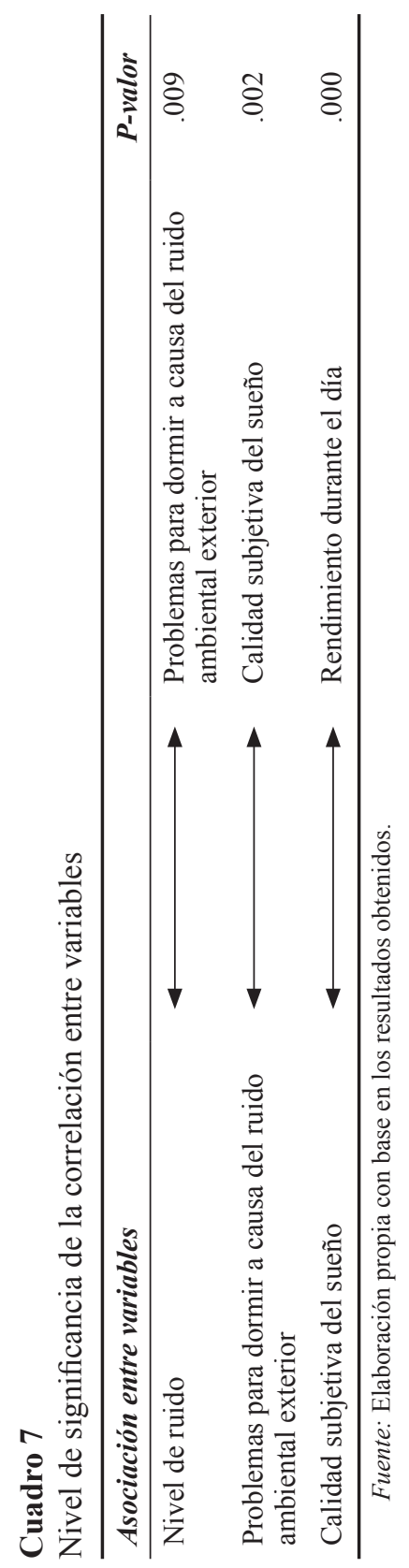


Por otro lado, los resultados indican que una de las respuestas más comunes entre los integrantes de la muestra que manifestaron problemas para dormir durante el último mes, respecto a las causas de dichos problemas, fue el ruido ambiental proveniente del exterior, específicamente el ruido vehicular, lo cual coincide con los hallazgos presentados por Öhrström, Hadzibajramovic, Holmes y Svensson (2006, p. 116).

Si bien en este caso las mediciones de ruido se realizaron durante horarios diurnos, esta respuesta se encuentra correlacionada significativamente con los niveles de ruido $\left(\mathrm{L}_{\mathrm{eq}}\right)$ identificados en el muestreo, con un p-valor de .009; de tal forma que, a mayor ruido de tráfico presente, más manifestaciones de problemas para dormir asociados al ruido ambiental. Esto puede deberse a que, como se explicó en el Cuadro 3, las vías seleccionadas son mayormente de uso comercial y alimentadora, por lo que cuentan con constante circulación vehicular las 24 horas del día.

Por otra parte, la variable de problemas para dormir a causa de ruido ambiental proveniente del exterior se encontró significativamente asociada a la variable de calidad subjetiva del sueño, con un p-valor de .002. Esto significa que, a mayor presencia de problemas para dormir por esta causa, la percepción de la calidad del sueño tiende a ser más negativa.

Puede afirmarse que, aun cuando los niveles de ruido identificados en el presente estudio no se encontraron directamente asociados a la calidad subjetiva del sueño, se presenta una asociación indirecta entre ambas variables de estudio, puesto que ambas se encuentran significativamente asociadas a la variable problemas para dormir a causa del ruido ambiental exterior. Por lo tanto, es correcto afirmar que, durante el último mes, el $45.2 \%$ de los integrantes de la muestra presentaron trastornos del sueño asociados al ruido ambiental por tráfico vehicular. Este resultado coincide con lo expuesto por De Santillana et al. (2010, p. 8), quienes señalan que los trastornos del sueño presentan una elevada prevalencia en la población de los países industrializados.

Por otro lado, aunque Álvarez (s.f.) establece que los efectos que el ruido ejerce sobre el comportamiento del individuo tienen que ver mayormente con su rendimiento, en el caso del presente estudio no fue posible establecer una correlación significativa entre las dos variables. Sin embargo, se presentó una correlación significativa entre el rendimiento y la calidad subjetiva del sueño, con un p-valor de .000 , lo cual significa que a mayor percepción negativa de la calidad del sueño, mayor presencia de problemas sobre el rendimiento diario. Este resultado coincide con lo expuesto por Quevedo y Quevedo (2011, p. 50), quienes consideran que la calidad del sueño es uno de los factores que se ligan directamente al desempeño del in- 
dividuo, y con Carskadon (1990, p. 5), quien identifica una relación entre el sueño insuficiente y la somnolencia diurna.

Considerando que la calidad subjetiva del sueño presentó una correlación significativa con la variable problemas para dormir a causa del ruido ambiental exterior, se puede inferir que ésta última se encuentra asociada indirectamente al rendimiento. En este sentido, el $26.8 \%$ de la muestra percibe un efecto negativo sobre su rendimiento diurno, lo cual es reconocido por la OMS (1999, pp. 4-5) como un efecto de índole psicológica.

\section{Conclusiones}

El ruido vehicular es un contaminante ambiental que genera problemas de salud, tanto física como psicológica, en las personas que se exponen a él de manera continua. Los pobladores de zonas urbanas resultan ser los más afectados ante este fenómeno, sobre todo cuando habitan cerca de vías que son altamente transitadas. En el caso de la ciudad de Matamoros, Tamaulipas, puede afirmarse que los niveles de ruido vehicular exceden los límites máximos permisibles establecidos por los organismos internacionales en materia de ruido ambiental.

Así mismo, queda en evidencia que el ruido vehicular identificado en los diferentes puntos de la ciudad se asocia a la calidad del sueño de los pobladores de la mancha urbana, y que la calidad del sueño se relaciona, a su vez, con el rendimiento de la población de estudio.

Por lo tanto, se considera que las estrategias preventivas deberían estar en función de:

1) La regulación pertinente y actualizada respecto a la emisión de ruido ambiental por vehículos automotores en circulación, lo que implicaría que las autoridades en la materia desarrollen estudios a largo plazo, e incluso que establezcan estaciones fijas de monitoreo.

2) La educación a la población sobre medidas preventivas para realizar las adaptaciones pertinentes en su hogar y protegerse del ruido exterior.

3) Promover el establecimiento de un programa de verificación continua de las condiciones de los vehículos automotores activos, sin importar el orden de procedencia, lo que serviría además como un censo real de las unidades que transitan. 
Por otro lado, es importante que las autoridades municipales, estatales $\mathrm{y}$ federales realicen estudios de ruido ambiental a largo plazo que aseguren el cumplimiento normativo, así como incluir tal cumplimiento en la agenda de las diferentes instituciones de salud de las áreas urbanas.

Por último, se considera pertinente la ampliación del estudio a otras zonas, así como la evaluación de los niveles de ruido en horarios vespertinos y nocturnos, que permita tener una mejor comprensión del comportamiento del ruido ambiental durante la noche.

Respecto al proyecto de ruido ambiental por tráfico vehicular, que originó el presente trabajo de investigación, se considera pertinente la continuación de trabajo de campo para obtener información en muestras más extensas y con tiempos de medición más amplios.

\section{Bibliografía}

Alfie, M. y Salinas, O. (2017). Ruido en la ciudad. Contaminación auditiva y ciudad caminable. Estudios Demográficos y Urbanos, 32(1), 65-96. Recuperado de http://estudiosdemograficosyurbanos.colmex.mx/index. php/edu/article/view/1613/pdf

Álvarez, T. (s.f.). Aspectos ergonómicos del ruido: evaluación (Documento de investigación). España: Instituto Nacional de Seguridad e Higiene en el Trabajo. Recuperado de http://www.insht.es/Ergonomia2/Contenidos/ Promocionales/Ruido\%20y\%20Vibraciones/ficheros/DTE-Aspectos ErgonomicosRUIDOVIBRACIONES.pdf

Blaivas, A. (2012). Información general sobre los trastornos del sueño. Página oficial de University of Maryland Medical Center, Estados Unidos. Recuperado de https://www.umms.org/ummc/patients-visitors/ health-library/medical-encyclopedia-spanish

Bobes, J. (1992). Los trastornos del sueño (insomnio). España: Rialp.

Carrillo, J. (1994). Dos décadas del sindicalismo en la industria maquiladora de exportación. Examen en las ciudades de Tijuana, Juárez y Matamoros. Ciudad de México: Universidad Autónoma Metropolitana, Unidad Iztapalapa / Miguel Ángel Porrúa

Carskadon, M. (1990). Patterns of sleep and sleepiness in adolescents. Pediatrician, 17, 5-12. Recuperado de http:/www.sleepforscience.org/ stuff/contentmgr/files/94a9f6f63ae18bc30f3d2d0b005e42fa/pdf/carska don_pediatrician1990.pdf

Centro Estatal de Vigilancia Epidemiológica y Control de Enfermedades (CEVECE). (s.f.) Efectos a la salud por ruido (Documento de investi- 
gación). México: Secretaría de Salud del Estado de México. Recuperado de http://salud.edomex.gob.mx/cevece/documentos/documentostec/ documentos/Efecs_ruido.pdf

Chávez, J. R. (2006). Ruido: efectos sobre la salud y criterio de su evaluación al interior de recintos. Ciencia y Trabajo, 8(20), 42-46. Recuperado de http://sgpwe.izt.uam.mx/files/users/uami/patt/3._Contaminacion_Fisica/ 3 _ruidoefectos.pdf

Chóliz, M. (1994). Emoción, activación y trastornos del sueño. Anales de Psicología, 10(2), 217-229. Recuperado de http://www.um.es/analesps/ v10/v10_2/11-10_2.pdf

Chóliz, M. (1999). Ansiedad y trastornos del sueño. En E. G. Fernández-Abascal y F. Palermo (eds.), Emociones y salud (pp.159-182). Barcelona: Ariel. De Santillana, S., Esquinca, J. L. García, A., Maldonado, J., Rodríguez, D. M., Torres, L. y Zamora, M. A. (2010). Diagnóstico y tratamiento de los trastornos del sueño (Documento de investigación). Ciudad de México: Centro Nacional de Excelencia Tecnológica en Salud, CENETEC. Recuperado de http://www.cenetec.salud.gob.mx/descargas/gpc/Cata logoMaestro/385_IMSS_10_Trastorno_sueno/EyR_IMSS_385_10.pdf Falleti, M. G., Maruff, P., Collie, A., Darby, D. G. y McStephen, M. (2003). Qualitive similarities in cognitive impairment associated with $24 \mathrm{~h}$ of sustained wakefulness and a blood alcohol concentration of $0.05 \%$. Journal of Sleep Research, 12(4), 265-274. Recuperado de https://online library.wiley.com/doi/epdf/10.1111/j.1365-2869.2003.00363.x

Frei, P., Mohler, E. y Röösli, M. (2014). Effect of nocturnal road traffic noise exposure and annoyance on objective and subjective sleep quality. International Journal of Hygiene and Environmental Health, 217(2-3), 188-195. Recuperado de https://www.sciencedirect.com/science/article/ pii/S1438463913000576?via\%3Dihub\#!

Hernández, A. (2006). Trastornos del sueño y su influencia en la conducta. Revista Biomédica Medwave, 6(2), e3361. Recuperado de: http://www. medwave.cl/link.cgi/Medwave/PuestaDia/Cursos/3361

Instituto Nacional de Ecología y Cambio Climático (INECC). (2010). Estudio de emisiones y actividad vehicular de Matamoros y Reynosa, Tamaulipas (Reporte final). Ciudad de México: Semarnat. Recuperado de https://www.gob.mx/cms/uploads/attachment/file/112405/2010_ CGCSA_RSD_Tamaulipas.pdf

Instituto Nacional de Estadística y Geografía (INEGI). (2015). Encuesta intercensal. Ciudad de México: Instituto Nacional de Estadística y Geografía. Recuperado de https://www.inegi.org.mx/programas/inter censal/2015/ 
International Organization for Standardization. (2007). Norma ISO 1996-2: 2007, Acoustics-Description, measurement and assessment of environmental noise. Part 2: Determination of environmental noise levels. Ginebra, Suiza: International Organization for Standardization.

International Organization for Standardization. (2016). Norma ISO 1996-1: 2016, Acoustics-description, measurement and assessment of environmental noise. Part 1: Basic quantities and assessment procedures. Ginebra, Suiza: International Organization for Standardization.

Martínez, A. (2005). Ruido por tráfico urbano: conceptos, medidas descriptivas y valoración económica. Revista de Economía y Administración, 2(1), 1-49. Recuperado de http://www.uao.edu.co/sites/default/files/ RUIDO 0.PDF

Medina, M. J. (2004). Influencia del trabajo nocturno y la rotación de turnos en el individuo. Ponencia presentada en VI Congreso Internacional de Ergonomía. Sociedad de Ergonomistas de México, A. C. / Universidad de Guanajuato. Guanajuato, México, 26 al 29 de mayo.

Medina, A., Feria, D. J. y Oscoz, G. (2009). Los conocimientos sobre el sueño y los cuidados enfermeros para un buen descanso. Enfermería Global, 17, 1-18. Recuperado de http://revistas.um.es/eglobal/article/ view/75151/72901

Morales, C. (2006). 300 millones de personas sufren de contaminación acústica en el mundo: el ruido deja en silencio al planeta. Ciencia y Trabajo, 8(20), 45-49. Recuperado de http://www.bvsde.paho.org/ bvsacd/cd65/ruido.pdf

Norma Oficial Mexicana - NOM-079-ECOL-1994. (1994). Norma Oficial Mexicana NOM-079-ECOL-1994, que establece los límites máximos permisibles de emisión de ruido de los vehículos automotores nuevos en planta y su método de medición. Ciudad de México: Secretaría de Medio Ambiente y Recursos Naturales. Recuperado de http://biblioteca.semar nat.gob.mx/janium/Documentos/Ciga/agenda/PPD02/DO2294n.pdf

Norma Oficial Mexicana - NOM-080-ECOL-1994. (1994). Norma Oficial Mexicana NOM-080-ECOL-1994, que establece los límites máximos permisibles de emisión de ruido proveniente del escape de los vehículos automotores, motocicletas y triciclos motorizados en circulación y su método de medición. Ciudad de México: Secretaría de Medio Ambiente y Recursos Naturales. Recuperado de http://biblioteca.semarnat.gob. $\mathrm{mx} /$ janium/Documentos/Ciga/agenda/PPD02/DO2295n.pdf

Norma Oficial Mexicana - NOM-081-ECOL-1994. (1994). Norma Oficial Mexicana NOM-081-ECOL-1994, que establece los límites máximos permisibles de emisión de ruido de las fuentes fijas y su método de 
medición. Ciudad de México: Secretaría de Medio Ambiente y Recursos Naturales. Recuperado de http://biblioteca.semarnat.gob.mx/janium/ Documentos/Ciga/agenda/PPD02/081.pdf

Norma Oficial Mexicana - NOM -011-STPS 2001. (2001). Norma Oficial Mexicana NOM-011-STPS-2001. Condiciones de seguridad e higiene en los centros de trabajo donde se genere ruido. Ciudad de México: Secretaría del Trabajo y Previsión Social. Recuperado de http://www. ordenjuridico.gob.mx/Documentos/Federal/wo69365.pdf

Observatorio de Salud y Medio Ambiente de Andalucía (OSMAN). (s.f.) Ruido y salud (Informe). España: Junta de Andalucía. Recuperado de https://www.diba.cat/c/document library/get file?uuid=72b1d2fd-c5e54751-b071-8822dfdfdded\&groupId $=7294824$

Öhrström, E., Hadzibajramovic, E., Holmes, M. y Svensson, H. (2006). Effects of road traffic noise on sleep: Studies on children and adults. Journal of Environmental Psychology, 26(2), 116-126. Recuperado de https:/www.sciencedirect.com/science/article/pii/S0272494406000375 Organización Mundial de la Salud (OMS). (1999). Guías para el ruido urbano (Documento de investigación). Ginebra, Suiza: Organización Mundial de la Salud. Recuperado de https://ocw.unican.es/pluginfile. php/965/course/section/1090/Guias\%2520para\%2520el\%2520ruido\% 2520urbano.pdf

Quevedo, V. y Quevedo, R. (2011). Influencia del grado de somnolencia, cantidad y calidad de sueño sobre el rendimiento académico en adolescentes. International Journal of Clinical and Health Psychology, 11(1), 49-65. Recuperado de http://www.aepc.es/ijchp/articulos_pdf/ijchp-370.pdf

Ramírez, A. y Domínguez, E. (2011). El ruido vehicular urbano: problemática agobiante de los países en vías de desarrollo. Revista de la Academia Colombiana de Ciencias Exactas, Físicas y Naturales, 35(137), 509-530. Recuperado de http://www.scielo.org.co/pdf/racefn/v35n137/v35n137 a09.pdf

Reglamento para la Prevención y Control de la Contaminación Ambiental Originada por la Emisión de Ruidos. (1976). Página oficial de la Secretaría de Gobernación. Ciudad de México Recuperado de http://dof.gob. $\mathrm{mx} /$ nota_detalle.php? codigo $=4912145 \&$ fecha $=02 / 01 / 1976$

Secretaría de Comunicaciones y Transportes. (1984). Norma de Servicios Técnicos: Proyecto Geométrico de Carreteras (Documento de trabajo, vol. 2.01.01). Ciudad de México. Recuperado de http://www.sct.gob. $\mathrm{mx} /$ fileadmin/DireccionesGrales/DGST/Normas/AAA4.pdf

Sierra, J. C., Jiménez, C. y Martín, J. D. (2002). Calidad del sueño en estudiantes universitarios: importancia de la higiene del sueño. Salud Men- 
tal, 25(6), 35-43. Recuperado de http://www.redalyc.org/pdf/582/5826 2505.pdf

Sygna, K., Aasvang, G., Aamodt, G., Oftedal, B. y Hjertager, N. (2014). Road traffic noise, sleep and mental health. Environmental Research, 131, 17-24. Recuperado de https://www.sciencedirect.com/science/article/ pii/S0013935114000371

Torres, V. y Monteghirfo, R. (2011). Trastornos del sueño. Archivos de Medicina Interna, 33(Supl.1), S01-S46. Recuperado de https://www.carmenaguilera-lorenzo.es/app/download/5786007362/Trastornos+del+sue $\% \mathrm{C} 3 \% \mathrm{~B} 1 \mathrm{o} . \mathrm{pdf}$

Velázquez, Y., Zamorano, B. y Ruiz, L. (2017). Siniestralidad vial en la frontera norte de Tamaulipas. Enfoque en los procesos administrativos de control. Estudios Fronterizos, 18(36), 1-24. Recuperado de http:// www.scielo.org.mx/pdf/estfro/v18n36/2395-9134-estfro-18-36-00001en.pdf

Zamorano, B., Peña, F., Parra, V., Velázquez, Y. y Vargas, J. I. (2015). Contaminación por ruido en el centro histórico de Matamoros. Acta Universitaria, 25(5), 20-27. Recuperado de http://www.scielo.org.mx/pdf/au/ v25n5/v25n5a3.pdf

\section{Acerca de los autores}

Benito Zamorano González es maestro en Ciencias de Salud Ocupacional, con formación profesional en prevención de riesgos. Actualmente es profesor investigador en la licenciatura de Seguridad, Salud y Medio Ambiente de la Universidad Autónoma de Tamaulipas, Unidad Académica Multidisciplinaria; es integrante del cuerpo académico Psicología y Prevención de Riesgos. Sus líneas de investigación son: psicología, prevención de riesgos y medio ambiente. ORCID: http://orcid.org/0000-0003-0422-3001

Entre sus trabajos recientes se encuentran:

Zamorano, B., Peña, F., Parra, V., Velázquez, Y. y Vargas, J. I. (2015). Contaminación por ruido en el centro histórico de Matamoros. Acta Universitaria, 25(5), 20-27.

Velázquez, Y., Zamorano, B. y Ruiz, L. (2017). Siniestralidad vial en la frontera norte de Tamaulipas. Enfoque en los procesos administrativos de control. Estudios Fronterizos, 18(36), 1-24.

Parra V., Vargas J. I., Zamorano, B., Peña, F., Velázquez, Y., Ruiz, L. y Monreal, Ó. (2016). Adicción y factores determinantes en el uso problemático del internet en una muestra de jóvenes universitarios. Edutec, 56, 60-73. 
Yolanda Velázquez Narváez es doctora en Educación, con formación profesional en prevención de riesgos. Actualmente es profesora investigadora en la licenciatura de Seguridad, Salud y Medio Ambiente de la Universidad Autónoma de Tamaulipas, Unidad Académica Multidisciplinaria (con 15 años de experiencia); es integrante del cuerpo académico Psicología, Cognición y Cultura. Sus líneas de investigación son: educación y prevención de riesgos. ORCID: http://orcid.org/0000-0001-5591-8474

Entre sus trabajos recientes se encuentran:

Velázquez, Y., Zamorano, B. y Ruiz, L. (2017). Siniestralidad vial en la frontera norte de Tamaulipas. Enfoque en los procesos administrativos de control. Estudios Fronterizos, 18(36), 1-24.

Zamorano, B., Peña, F., Parra, V., Velázquez, Y. y Vargas, J. I. (2015), Contaminación por ruido en el centro histórico de Matamoros. Acta Universitaria, 25(5), 20-27.

Parra V., Vargas J. I., Zamorano, B., Peña, F., Velázquez, Y., Ruiz, L. y Monreal, Ó. (2016). Adicción y factores determinantes en el uso problemático del internet en una muestra de jóvenes universitarios. Edutec, 56, 60-73.

Fabiola Peña Cárdenas es doctora en Psicología Aplicada, con formación profesional en psicología. Actualmente es profesora investigadora en la licenciatura de Psicología de la Universidad Autónoma de Tamaulipas, Unidad Académica Multidisciplinaria (con 15 años de experiencia); es integrante del cuerpo académico Psicología, Cognición y Cultura. Sus líneas de investigación son: psicología y prevención de riesgos. ORCID: https://orcid. org/0000-0001-5895-0816

Entre sus trabajos recientes se encuentran:

Monreal, Ó., Velázquez, Y., Peña, F., Ruiz, L., Zamorano, B., Parra, V. y Vargas J. I. (2016). Rol laboral como factor de riesgo psicosocial en los profesionales de la salud. Gestión Práctica de Riesgos Laborales, 137, 16-27.

Zamorano, B., Peña, F., Parra, V., Velázquez, Y. y Vargas, J. I. (2015). Contaminación por ruido en el centro histórico de Matamoros. Acta Universitaria, 25(5), 20-27.

Parra V., Vargas J. I., Zamorano, B., Peña, F., Velázquez, Y., Ruiz, L. y Monreal, Ó. (2016). Adicción y factores determinantes en el uso problemático del internet en una muestra de jóvenes universitarios. Edutec, 56, 60-73.

Lucía Ruiz Ramos es doctora en Psicología Aplicada, con formación profesional en psicología. Actualmente es profesora investigadora en la licen- 
ciatura de Psicología de la Universidad Autónoma de Tamaulipas, Unidad Académica Multidisciplinaria (con 15 años de experiencia); es integrante del cuerpo académico Psicología, Cognición y Cultura. Sus líneas de investigación son: psicología y prevención de riesgos. ORCID: http://orcid. org/0000-0002-7122-6758

Entre sus trabajos recientes se encuentran:

Monreal, Ó., Velázquez, Y., Peña, F., Ruiz, L., Zamorano, B., Parra, V. y Vargas J. I. (2016). Rol laboral como factor de riesgo psicosocial en los profesionales de la salud. Gestión Práctica de Riesgos Laborales, 137, 16-27.

Velázquez, Y., Zamorano, B. y Ruiz, L. (2017). Siniestralidad vial en la frontera norte de Tamaulipas. Enfoque en los procesos administrativos de control. Estudios Fronterizos, 18(36), 1-24.

Parra V., Vargas J. I., Zamorano, B., Peña, F., Velázquez, Y., Ruiz, L. y Monreal, Ó. (2016). Adicción y factores determinantes en el uso problemático del internet en una muestra de jóvenes universitarios. Edutec, 56, 60-73.

Óscar Monreal Aranda es doctor en Psicología Aplicada, con formación profesional en psicología. Actualmente es profesor investigador en la licenciatura de Psicología de la Universidad Autónoma de Tamaulipas, Unidad Académica Multidisciplinaria; es integrante del cuerpo académico Psicología y Prevención de Riesgos. Sus líneas de investigación son: psicología y prevención de riesgos. ORCID: https://orcid.org/0000-0003-2039-6375

Entre sus trabajos recientes se encuentran:

Monreal, Ó., Velázquez, Y., Peña, F., Ruiz, L., Zamorano, B., Parra, V. y Vargas J. I. (2016). Rol laboral como factor de riesgo psicosocial en los profesionales de la salud. Gestión Práctica de Riesgos Laborales, 137, $16-27$.

Ruiz, L., Velázquez, Y., Monreal, Ó, Peña, F., Vargas, J. I. y Hernández, I. (2016). Prevalencia del nivel de estrés académico en universitarios, con diferencia de género. Paraninfo Digital, 10(25), 1-5.

Parra V., Vargas J. I., Zamorano, B., Peña, F., Velázquez, Y., Ruiz, L. y Monreal, Ó. (2016). Adicción y factores determinantes en el uso problemático del internet, en una muestra de jóvenes universitarios. Edutec, 56, 60-73.

Víctor Parra Sierra es maestro en Comunicación Académica, con formación profesional en sistemas. Actualmente es profesor investigador en las licenciaturas de Psicología, Enfermería y Seguridad, Salud y Medio Ambiente de 
la Universidad Autónoma de Tamaulipas, Unidad Académica Multidisciplinaria; es integrante del cuerpo académico Psicología y Prevención de Riesgos. Sus líneas de investigación son: psicología y prevención de riesgos. ORCID: http://orcid.org/0000-0002-3877-9713

Entre sus trabajos recientes se encuentran:

Monreal Ó., Velázquez, Y., Peña, F., Ruiz, L., Zamorano, B., Parra, V. y Vargas, J. I. (2016). Rol laboral como factor de riesgo psicosocial en los profesionales de la salud. Gestión Práctica de Riesgos Laborales, 137, 16-27.

Parra V., Vargas J. I., Zamorano, B., Peña, F., Velázquez, Y., Ruiz, L. y Monreal, Ó. (2016). Adicción y factores determinantes en el uso problemático del internet en una muestra de jóvenes universitarios. Edutec, 56, 60-73.

José Ignacio Vargas Martínez es maestro en Ciencias de Enfermería, con formación profesional en enfermería. Actualmente es profesor investigador en la licenciatura en Enfermería de la Universidad Autónoma de Tamaulipas, Unidad Académica Multidisciplinaria; es integrante del cuerpo académico Psicología y Prevención de Riesgos. Sus líneas de investigación son: salud y prevención de riesgos. ORCID: https://orcid.org/0000-0003-2467-5806

Entre sus trabajos recientes se encuentran:

Monreal, Ó., Velázquez, Y., Peña, F., Ruiz, L., Zamorano, B., Parra, V. y Vargas, J. I. (2016). Rol laboral como factor de riesgo psicosocial en los profesionales de la salud. Gestión Práctica de Riesgos Laborales, 137, $16-27$.

Ruiz, L., Velázquez, Y., Monreal, Ó, Peña, F., Vargas, J. I. y Hernández, I. (2016). Prevalencia del nivel de estrés académico en universitarios, con diferencia de género. Paraninfo Digital, 10(25), 1-5.

Parra V., Vargas J. I., Zamorano, B., Peña, F., Velázquez, Y., Ruiz, L. y Monreal, Ó. (2016). Adicción y factores determinantes en el uso problemático del internet en una muestra de jóvenes universitarios. Edutec, 56, 60-73.

Recepción: 5 de abril de 2017.

Aceptación: 24 de abril de 2018. 
